\title{
TA fluorescence detector calibration by UV LED with an unmanned aerial vehicle
}

\author{
Yuichiro Tameda* \\ Osaka Electro-Communication University, Neyagawa, Osaka, Japan \\ E-mail: tameda@osakac.ac.jp
}

\section{Takayuki Tomida}

Shinshu University, Nagano, Nagano, Japan

E-mail: tomida@shinshu-u.ac.jp

\section{Motoki Hayashi}

Shinshu University, Nagano, Nagano, Japan

E-mail: 15tm524jeshinshu-u.ac.jp

\section{Terutsugu Seki}

Shinshu University, Nagano, Nagano, Japan

E-mail: 17w2049h@shinshu-u.ac.jp

\section{for the Telescope Array Collaboration}

\begin{abstract}
We are developing a fluorescence detector (FD) calibration device called as Opt-copter which consists of an UV LED, a high accuracy GPS and an unmanned aerial vehicle (UAV). Opt-copter is a standard light source to be used to calibrate the Telescope Array (TA) FDs. The primary characteristic of Opt-copter is its high portability which enables us to put the light source on any position we want in the F.O.V. of FDs. Our upgraded UAV, has improved flight reliability and payload capacity. The calibration procedure is established by a test observation at the TA FD site. We evaluated the flight accuracy and developed a method to measure the FD geometry. We will report the current status of Opt-copter.
\end{abstract}

35th International Cosmic Ray Conference - ICRC2017

10-20 July, 2017

Bexco, Busan, Korea

* Speaker. 


\section{Introduction}

Telescope Array (TA) is the largest cosmic ray observatory in the northern hemisphere to observe ultra high energy cosmic rays, located in the western desert in Utah, U.S.A [1][2]. TA is a hybrid detector and consists of fluorescence detectors (FDs) and a surface detector (SD) array. FDs can measure the energies of cosmic rays calorimetrically detecting fluorescence UV light emitted from Nitrogen molecules excited by high energy particles of extensive air showers (EASs). Moreover, FDs can measure longitudinal developments of EASs which are very important to identify the particle type of primary cosmic rays.

The energies measured by two different FDs of TA are different at the level of $4 \pm 2 \%$, though this is consistent within the systematic error. It is important to separate the FD detection efficiency and atmospheric transparency to improve the FD systematic error. On the site, the FD absolute detection efficiency is monitored by various devices, such as YAP and Xe flasher which are mounted on each FD. However, we cannot compare the FD detection efficiency directly. This is the motivation to develop Opt-copter which is a standard light source mounted on an UAV and can fly in the F.O.V. of FDs. Using Opt-copter, we can measure the FD detection efficiency using a single light source. Additionally, Opt-copter also has the possibility to measure the geometry of FDs actively, compared with the passive method of star tracking analysis.

In this paper, we will report the specification of Opt-copter, the detail of its position measurement and the method of the FD telescope geometry measurement.

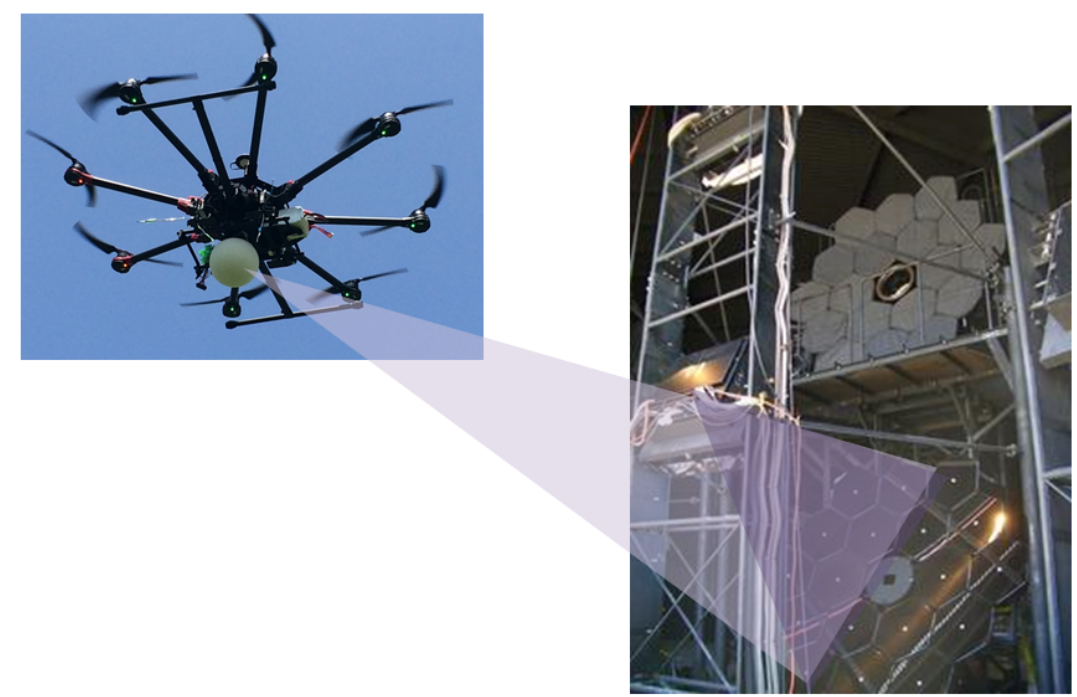

Figure 1: The diagram of FD calibration concept using Opt-copter. UAV with a LED light flasher is flying to the F.O.V. of TA FD. 


\section{Opt-copter}

Opt-copter is one of the calibration devices developed for TA FDs [3]. Figure 1 is the schematic view of the calibration by Opt-copter (Auger has also already developed a similar device [4]). Optcopter consists of a UAV, a high accuracy GPS system and a UV LED light source.

\subsection{UAV}

We use an eight rotor multicopter (S1000+, DJI), as shown in Fig. 2 (Left). The size of the multicopter is $400 \mathrm{~mm}$ (width) $\times 400 \mathrm{~mm}$ (depth) $\times 500 \mathrm{~mm}$ (height) when the arms are folded, so that it is easy to transfer. The weight is $4.4 \mathrm{~kg}$ with a LiPo battery and the maximum load weight is $6.6 \mathrm{~kg}$. The flight time is $15 \mathrm{~min}$. with a LiPo battery of $15000 \mathrm{mAh}$ when the total weight is 9.5 $\mathrm{kg}$. The accuracy of flight control is $\pm 1.5 \mathrm{~m}$. We operate Opt-copter only with a wind speed less than $5 \mathrm{~m} / \mathrm{s}$.

\subsection{GPS}

The required positioning accuracy is $\pm 0.1 \mathrm{~m}$ at $100 \mathrm{~m}$ apart from FDs. Therefore, we measure the position of Opt-copter by Real Time Kinematics (RTK) system using Piksi (SwiftNavigation), as shown in Fig. 2 (Middle). The accuracy of relative distance between the two GPS modules which are called base and rover by RTK system should be $\sim \mathrm{cm}$. According to our accuracy test in static situation, the relative distance between the two GPS modules is determined to be within 0.1 m.

\subsection{UV LED light source}

UV-LEDs (Roithner Lasertechnik, H2A1-H375-E) with a peak wavelength of $375 \mathrm{~nm}$ are used as a light source. The light source consists of 12 LEDs mounted on each side of the dodecahedron, as shown in Fig. 2 (Right). It is covered with a spherical diffuser made of acrylic resin to improve the uniformity of the light intensity. The stability of the light intensity is $\sim 0.1 \%$ measured in the laboratory. The temperature sensor is also implemented on Opt-copter and we also are planning an on flight shot-by-shot monitor of the light intensity. The azimuthal dependence of the light intensity is $\pm 9 \% / 2 \pi$ and $0.5 \sim 4 \%$ per degrees which is good enough, considering the flight stability of \pm 7 degrees.
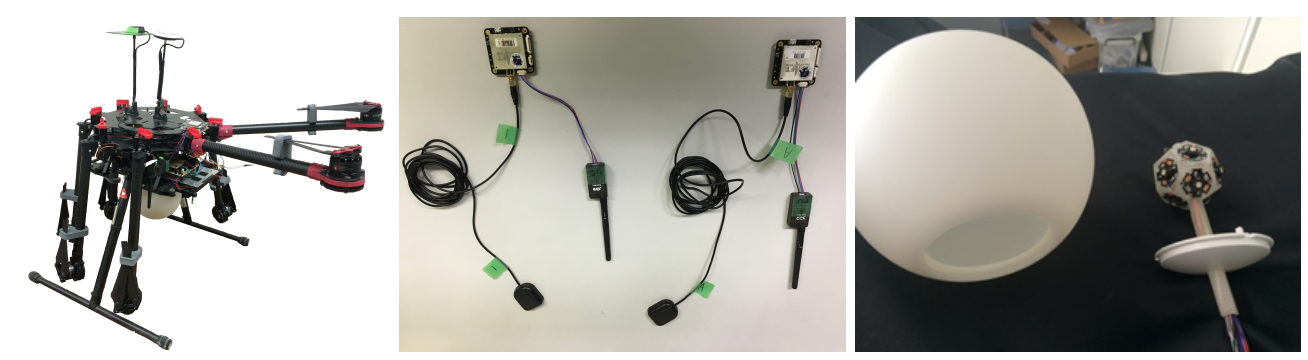

Figure 2: (Left) The eight rotor multicopter (S1000+, DJI) mounting the LED flasher under the body. The arms can be folded. (Middle) High accuracy GPS. One is installed at the well known point as base and the other is mounted on Opt-copter. (Right) UV-LEDs installed on the dodecahedron which will be covered by the spherical diffuser. 


\section{Flight position measurement}

The accuracy of the position measurement is \pm 4 and $\pm 6 \mathrm{~cm}$ in the horizontal and vertical direction measured on the ground in static situation. We performed flight test at the TA FD site on the 3rd and 6th of January, 2017. We flew Opt-copter $200 \mathrm{~m}$ from the center of the FD mirrors. The target position is the center of the F.O.V. of a standard PMT which is used for PMT gain monitoring. While the test flight, we vetoed the hybrid trigger not to send the trigger information to TA SD. We acquired the data with FD by normal trigger or an external trigger of 10 PPS. It was $-5^{\circ} \mathrm{C}$ so that the flight control, especially for height, became worse due to low temperature. Figure 3 is the comparison plot of azimuth angle of the center of gravity calculated from the sum of FADC values of each channel and projected one of the position measured by RTK system. The RTK position looks smooth, on the other hand the center of gravity looks like a step function. This is because the angular resolution of FD is one degree. Moreover, one should take into account the non-uniformity of PMT gain and the gap of PMTs.

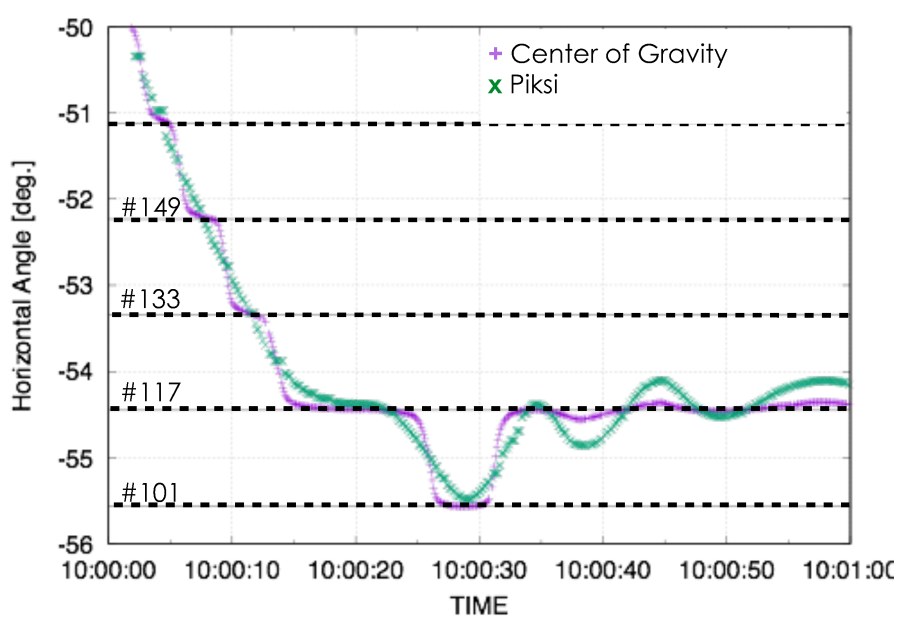

Figure 3: The time series of the horizontal angle in the F.O.V. of TA FD. (+) is the center of gravity calculated by FADC sum. $(\times)$ is GPS position measured by RTK system. Dotted lines are the center of PMT of \#149, 133, 117, 101.

We tried to check whether the RTK position is consistent or not with the FD center of gravity using two assumptions. One assumption is that when Opt-copter is flying on the edge of a PMT F.O.V., the center of gravity should be the same as the RTK position as shown in the left of Fig. 4. In the right panel of Fig. 4, when $a$ is at a minimum, Opt-copter is on the edge of PMT and $A$ is the uncertainty of the position. The other assumption is that when Opt-copter is on the edge of PMT F.O.V., the sum of FADC values should be the local minimum. In the right of Fig. 4, when the sum of FADC values is at a local minimum, Opt-copter is on the edge of the PMTs, and $B$ is the uncertainty of the position. $\Delta b$ is the difference between the minimum and the second minimum point so that this parameter is the systematic uncertainty of this assumption. The minimum of the FADC sum can be between the minimum and the second minimum point.

Table 1 shows the comparison of uncertainties of position measurement. The worst value of parameter $\mathrm{A}$ is $0.075^{\circ}$ corresponding to $26 \mathrm{~cm}$ and that of $\mathrm{B}$ is $0.017^{\circ}$. The accuracy of flight 

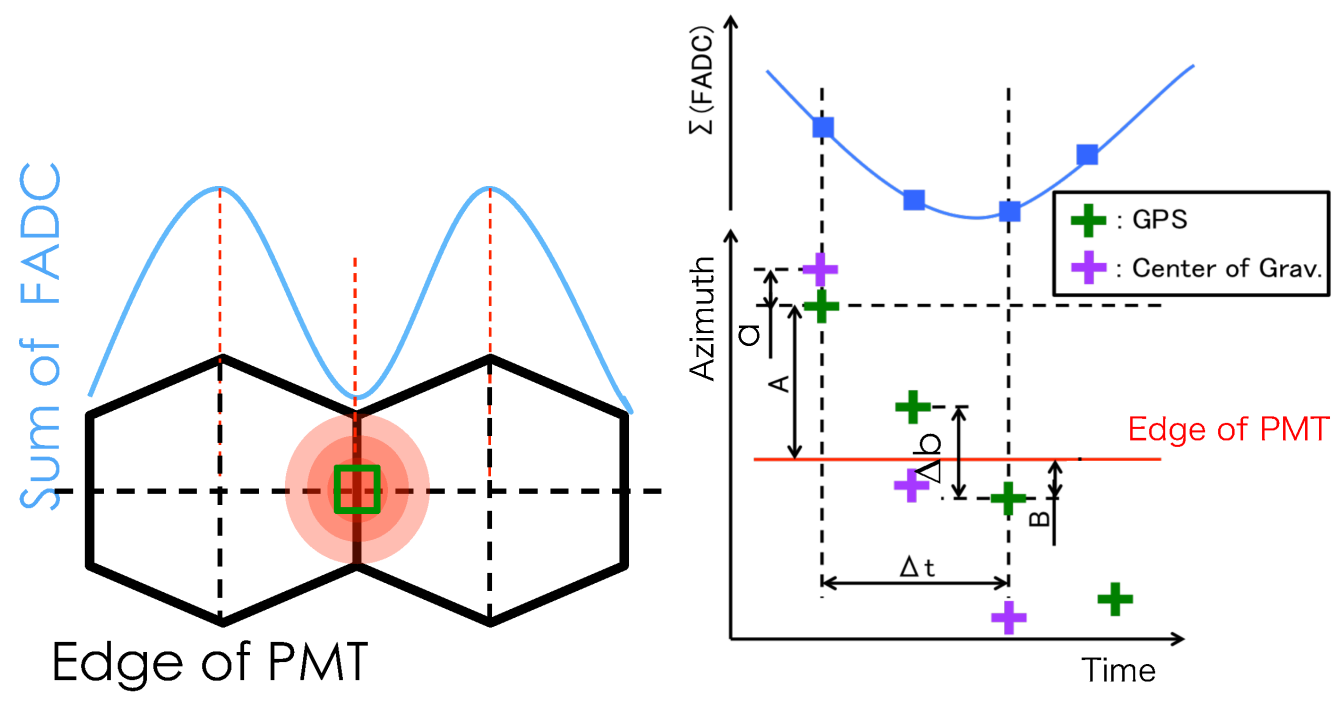

Figure 4: (Left) The concept of the analysis of the accuracy of flight position measurement. When the spot of Opt-copter is on the edge of the PMTs shown as filled circles, the center of gravity calculated by the sum of FADC values should be also on the edge of the PMTs and the sum of FADC values should be at a local minimum. (Right) Diagram of the parameters of $A, a, B$, and $\Delta b$ to estimate the uncertainty of position measurement.

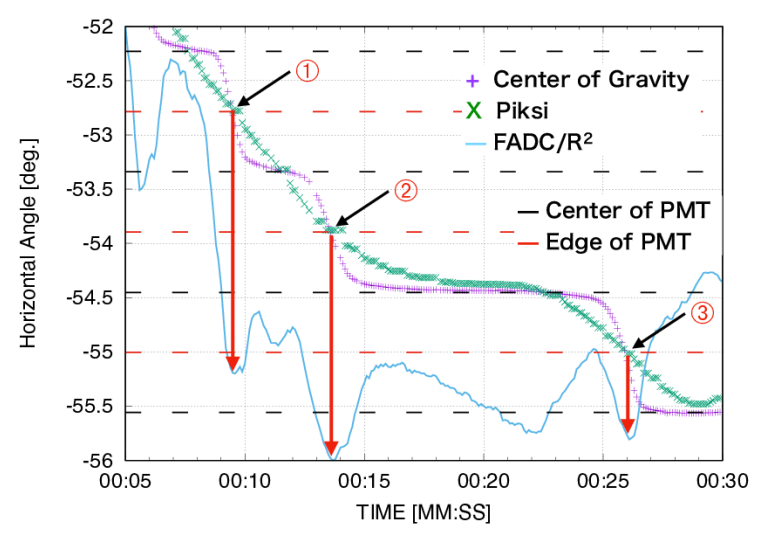

Figure 5: Zoomed plot of Fig. 3 and the sum of FADC values corrected by the distance $R$ between Optcopter and the FD mirror. Red dotted lines are the edges of PMT.

position is at least less than $26 \mathrm{~cm}$ as estimated by using TA FD data. 
Table 1: The uncertainties estimated by the two assumptions.

\begin{tabular}{r|r|r|r|r|} 
& $A$ [deg.] & $a$ [deg.] & $B$ [deg.] & $\Delta b$ [deg.] \\
\hline (1) & 0.019 & 0.001 & 0.017 & 0.002 \\
\hline (2) & 0.075 & 0.003 & 0.005 & 0.005 \\
\hline (3) & 0.030 & 0.010 & 0.016 & 0.001 \\
\hline
\end{tabular}

\subsection{Accuracy of flight control}
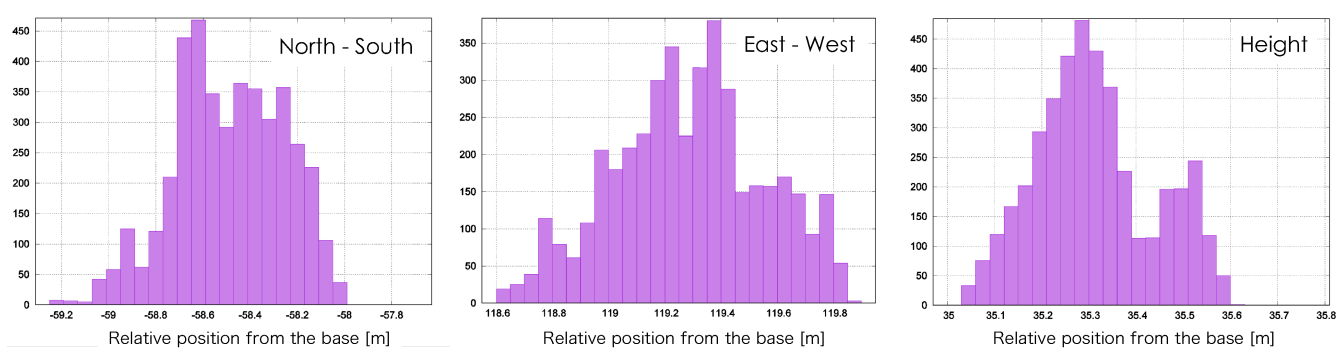

Figure 6: The histograms relative position between the surveyed point and Opt-copter in each dimensions.

The accuracy of flight control of S1000+ is $\pm 1.5 \mathrm{~m}$. Figure 6 shows the histogram of the relative position of Opt-copter to the surveyed point measured by RTK system while hovering. The hovering accuracy is $0.4 \mathrm{~m}$ in horizontal and $0.1 \mathrm{~m}$ in vertical as summarized in Table 2. However the difference between Opt-copter and the target position are $1 \mathrm{~m}$ and $5 \mathrm{~m}$ in horizontal and vertical, respectively. The difference of vertical direction is especially worse compared with the specification. The outdoor temperature was less than $-5^{\circ} \mathrm{C}$ while the test flight. The working environment temperature is $-10 \sim+40^{\circ} \mathrm{C}$. Below this temperature, the accuracy of flight control can become worse due to the pressure sensor for the flight controller. We should correct the temperature dependence under such cold condition.

Table 2: The comparison of the target position and average, maximum ,minimum and standard deviation of measured position of Opt-copter.

\begin{tabular}{l|r|r|r} 
& N-S [m] & E-W [m] & Height [m] \\
\hline Target position & -58.0 & 118.4 & 40.0 \\
\hline Measured position & -58.5 & 119.3 & 35.3 \\
\hline$\sigma$ & 0.24 & 0.37 & 0.13 \\
\hline Maximum & -58.0 & 119.9 & 35.6 \\
\hline Minimum & -59.3 & 118.6 & 35.0
\end{tabular}

\section{Spot size measurement}

The left panel of Fig. 7 shows the comparison plot of the angular distance of the center of gravity of FADC sum and RTK position from the center of the PMTs. If the angular resolution is quite good, the angular distances of the RTK position and the center of gravity are equal. However, 
if the resolution is about one degree, like TA FD, the center of gravity does not change much while a spot is inside a PMT, and when a spot is spreading to the next PMT, the center of gravity will start changing. We tried to estimate the spot size at the focal plane of FD using this characteristic. The schematic view of spot size estimation is shown in the right of Fig. 7. The spot of Opt-copter is spreading on the PMT at the focal plane as shown in Fig. 7(right). The radius of the spot can be calculated as $r_{\text {spot }}=\theta_{\text {PMTedge }}-\theta_{s}, \theta_{\text {PMTedge }}=0.5$ [degree]. For the PMT \#117 of telescope \#07 at the Black Rock Mesa (BRM) FD site, the diameter of the spot is 0.3 degree corresponding to 1.6 $\mathrm{cm}$. We are planning to investigate other telescopes.
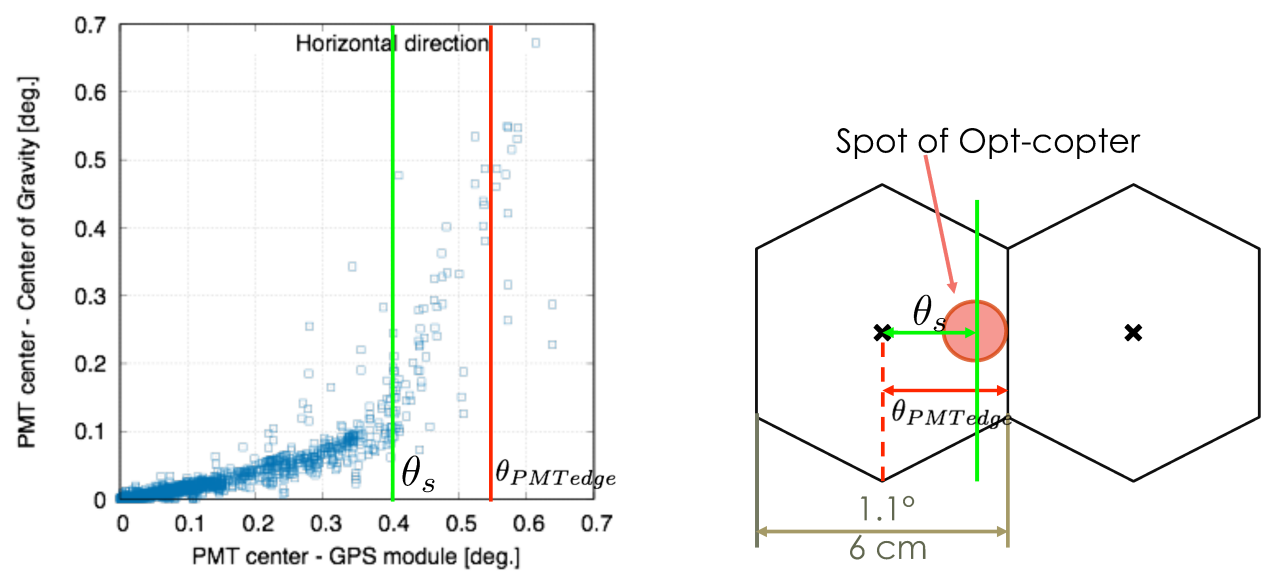

Figure 7: (Left) The angular distance of the RTK position and the center of gravity of FADC sum to the center of PMT for the PMT \#117, telescope \#07. (Right) The schematic view of how to estimate the spot size.

\section{Summary}

We are developing Opt-copter as a calibration device for TA FD. Opt-copter consists of an UV LED light source, a high accuracy GPS and a UAV. The accuracy of the position measurement is \pm 4 and $\pm 6 \mathrm{~cm}$ in the horizontal and vertical direction in static situation. We also estimate the accuracy of flight position measurement using TA FD data, and it is less than $26 \mathrm{~cm}$ which is consistent with the accuracy measured in static situation. The hovering accuracy of Opt-copter is $0.4 \mathrm{~m}$ in horizontal and $0.1 \mathrm{~m}$ in vertical. We also found that the difference between the hovering height and the target position becomes larger when it is less than $-10^{\circ} \mathrm{C}$ due to the temperature dependence of the pressure sensor. We tried to estimate the spot size and it is $1.6 \mathrm{~cm}$ for the PMT \#117 of the telescope \#07 at the BRM site. Opt-copter has a potential to measure the geometry of TA FD by measuring the edges and the spot sizes for each PMT. Therefore, we plan to fly Opt-copter in the F.O.V. of all TA FDs from August 2017.

\section{Acknowledgments}

The Telescope Array experiment is supported by the Japan Society for the Promotion of Science through Grants-in-Aid for Scientific Research on Specially Promoted Research (21000002) 
"Extreme Phenomena in the Universe Explored by Highest Energy Cosmic Rays" and for Scientific Research (19104006), and the Inter-University Research Program of the Institute for Cosmic Ray Research; by the U.S. National Science Foundation awards PHY-0307098, PHY-0601915, PHY-0649681, PHY-0703893, PHY-0758342, PHY-0848320, PHY-1069280, PHY-1069286, PHY1404495 and PHY-1404502; by the National Research Foundation of Korea (2015R1A2A1A01006870, 2015R1A2A1A15055344, 2016R1A5A1013277, 2007-0093860, 2016R1A2B4014967); by the Russian Academy of Sciences, RFBR grant 16-0200962a (INR), IISN project No. 4.4502.13, and Belgian Science Policy under IUAP VII/37 (ULB). The foundations of Dr. Ezekiel R. and Edna Wattis Dumke, Willard L. Eccles, and George S. and Dolores Doré Eccles all helped with generous donations. The State of Utah supported the project through its Economic Development Board, and the University of Utah through the Office of the Vice President for Research. The experimental site became available through the cooperation of the Utah School and Institutional Trust Lands Administration (SITLA), U.S. Bureau of Land Management (BLM), and the U.S. Air Force. We appreciate the assistance of the State of Utah and Fillmore offices of the BLM in crafting the Plan of Development for the site. We also wish to thank the people and the officials of Millard County, Utah for their steadfast and warm support. We gratefully acknowledge the contributions from the technical staffs of our home institutions. An allocation of computer time from the Center for High Performance Computing at the University of Utah is gratefully acknowledged.

\section{References}

[1] H.Tokuno, et al., New air fluorescence detectors employed in the Telescope Array experiment, NIM A, 676 (2012) 54-65

[2] T.Abu-Zayyad, et al., The surface detector array of the Telescope Array experiment, NIM A, 689 (2012) 87-97

[3] M. Hayashi, et al., Calibration of a fluorescence detector using a flying standard light source for the Telescope Array observatory, in proceedings of The 34th International Cosmic Ray Conference, POS ( ICRC2015) 692 (2015)

[4] F. Werner, Design and Test of a Flying Light Source for the Calibration of the Auger Fluorescence Telescopes, Thesis, Karlesruhe Institute of Technology (2010) 